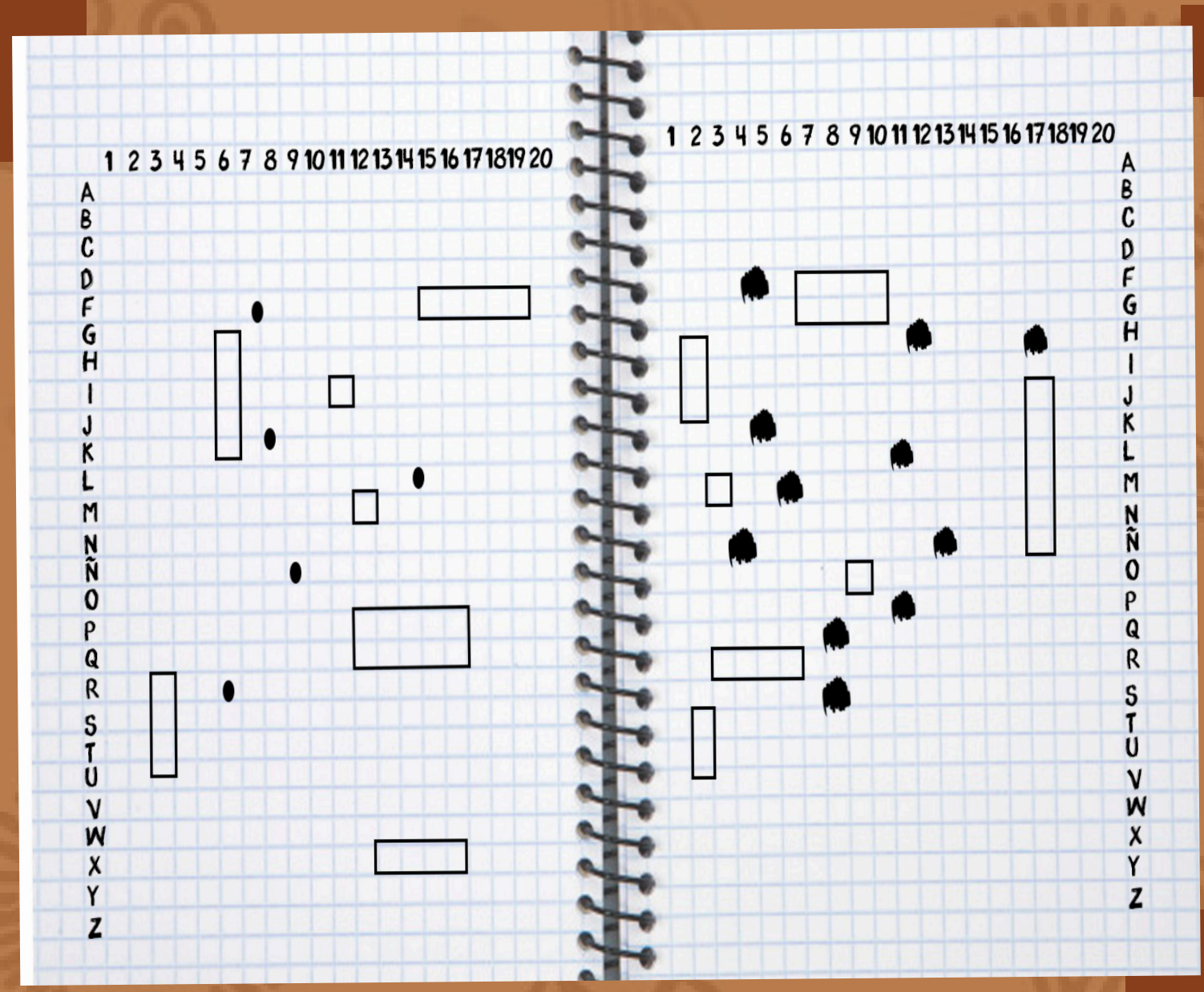

\title{
Los juegos en papel. Una manifestación de la cultura escolar Nicaragüense
}




\section{Los juegos en papel. Una manifestación de la cultura escolar Nicaragüense}

\section{The games on paper. a manifestation of Nicaraguan school culture}

\author{
Arlen Idania López Delgado \\ Licenciada en Pedagogía con mención en Educación \\ Primaria \\ ID Orcid: https://orcid.org/0000-0001-6269-3429 \\ arlenidania0685@hotmail.com
}

Recibido: 26-04-2021

Aceptado: 21-05-2021

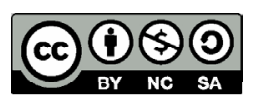

Copyright $\odot 2021$ UNAN-Managua Todos los Derechos Reservados.

\section{Yesika Karina Hernández Navarro}

Licenciada en Ciencias de la Educación

con mención en Ciencias Sociales.

ID Orcid: https://orcid.org/0000-0002-5344-1315 hernandezjesika569@gmail.com

\section{Resumen}

La escuela además de ser una institución social en donde se gesta la enseñanza de las ciencias y la formación ciudadana, es un espacio de socialización y constructor de culturas en la medida en que la comunidad escolar interrelaciona entre sí y con su medio. Este punto de partida conllevó a profesores investigadores a caracterizar los juegos en papel como una expresión cultural emergida en el aula de clase al margen de los procesos educativos, haciendo énfasis en identificar los tipos de juegos en papel, describir cómo se jugaban, determinar en qué momentos se jugaban y precisar los usos didácticos que estos tienen en los procesos educativos. La investigación se desarrolló mediante la metodología de la historia oral a través de talleres, entrevistas y encuestas, siendo sus participantes cinco grupos de clases de estudiantes. Con base en este proceso metodológico de investigación se lograron recopilar juegos como el ahorcado, el camino del diablo, rayos del sol, stop, juego de la $Z$, guerra naval, puntito, equis y cero, carrito y viendo el futuro. Finalmente, el artículo concluye haciendo una reflexión alrededor de los retos que han de asumir educadores e investigadores en el rescate de las manifestaciones culturales escolares que la modernidad ha ido relegada en el agitado diario vivir

Palabras claves: Cultura, Educación, Juegos en papel, Nicaragüense

\section{Abstract}

The school, besides being a social institution where the teaching of science and citizenship education takes place, is a space for socialization and a builder of cultures to the extent that the school community interrelates among itself and with its environment. This starting point led the teacher-researchers to characterize paper games as a cultural expression that emerged in the classroom outside the educational processes, with emphasis on identifying the types of paper games, describing how they were played, determining at what moments they were played and specifying the didactic uses they have in the educational processes. The research was developed by means of oral history methodology through workshops, interviews and surveys, with five groups of student classes as participants. Based on this methodological research process, games such as hangman, devil's path, sun rays, stop, Z game, naval war, dot, $x$ and zero, cart and seeing the future were collected. Finally, the article concludes with a reflection on the challenges that educators and researchers have to assume in the rescue of school cultural manifestations that modernity has relegated in the hectic daily life.

Keywords: Culture, Education, Paper games, Nicaraguan 


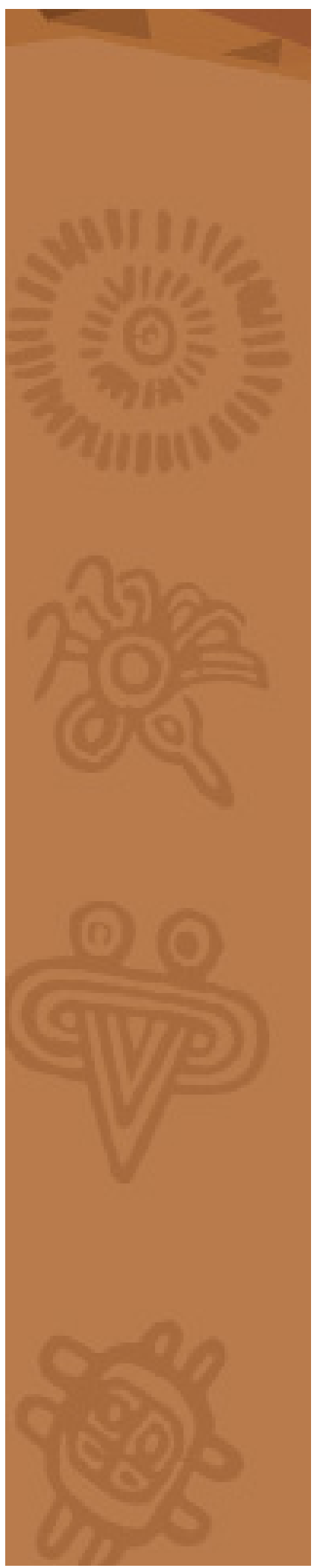

\section{Introducción}

La escuela es un espacio físico de interacción de personas en donde se gesta la enseñanza de las ciencias y se certifican las capacidades que los estudiantes van adquiriendo progresivamente en su formación ciudadana, sin embargo, más allá del rol formador que estrictamente compromete a todas las institución de Educación, la escuela también es un espacio de socialización y de construcción de culturas en donde los estudiantes desarrollan prácticas cotidianas que se manifiestan en sus códigos lingüísticos, formas de interacción, costumbres, ocios, recurrencias, entre otros, y en este caso, los juegos en papel no están exentos de la misma.

En la cultura tradicional nicaragüense suelen ser comunes los reencuentros de generaciones de estudiantes que compartieron grados y modalidades escolares, estos se han ido constituyendo como espacios de socialización en donde reaparecen entre algarabías y carcajadas aquellas anécdotas, recuerdos, curiosidades y relatos de momentos jocosos y pesarosos que formaron parte de la historia de vida de su época, y en todos ellos se pueden observar rasgos culturales que, con el pasar del tiempo unos siguen presentes aún en las generaciones presentes, y otros se han disipado y únicamente siguen alojados en la memoria colectiva de sus protagonistas.

Esto ha llevado a este equipo de investigadores a realizar una investigación de carácter social enfocada específicamente en el rescate de los juegos en papel presentes en las culturas escolares, esto como un aporte a la cultura nicaragüense y en aras de rescatar esos valiosos espacios escolares constructores de cultura que la modernidad, la tecnología y las cotidianidades de la vida silenciosamente ha ido confinando en lejanos recuerdos, pero que permanecen vivos en aquellas generaciones que no necesitaban condiciones sofisticadas para jugar con sus compañeros de clases.

En este sentido, el presente artículo en su ámbito general se enfoca en caracterizar los tipos de juegos en papel presentes en las culturas escolares, y en sus acciones específicas se ha enfatizado en identificar los tipos de juegos en papel, describir cómo se jugaban, determinar en qué momentos se jugaban y precisar los usos didácticos que estos tienen en los procesos educativos. En vista de esto, en el transcurso del escrito se describe la metodología utilizada y un conjunto de diez juegos en papel que han sido recopilados durante el proceso investigativo a través de la implementación de la metodología de la historia oral.

Asimismo, es oportuno señalar que los estudios de esta naturaleza representan un aporte significativo para el rescate y promoción de la cultura nicaragüense, y cabe destacar que esta investigación es continuidad de otros procesos investigativos que se han llevado a cabo dentro de esta comunidad de investigadores nicaragüenses, entre ellos Promoviendo los juegos tradicionales nicaragüenses. Una experiencia de animación sociocultural (Díaz, Arauz, Sandoval y Rizo, 2019) y Los castigos escolares en la década de los setenta. Relatos de estudiantes y profesores (Cruz, Díaz y Hernández, 2020), en donde se encuentran resultados significativos que vienen a aportar a la construcción de la cultura nicaragüense. 


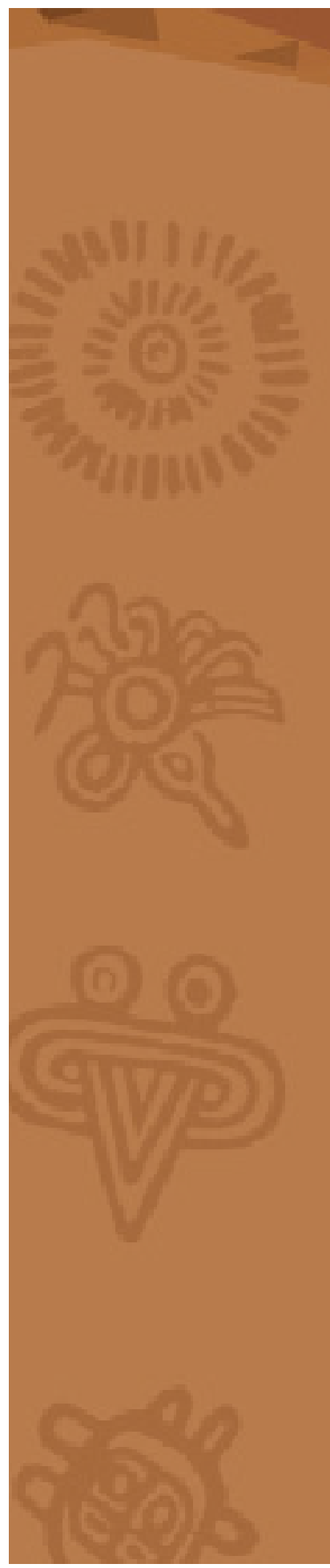

\section{MATERIALES Y MÉTODOS}

\section{Investigación histórica}

En Bernal (2010) se expresa que la investigación histórica se caracteriza por estudiar y examinar los fenómenos y sucesos del pasado, para así determinar su relación con el presente y con el futuro. También el autor sostiene que, en este tipo de investigación cualitativa, las fuentes y técnicas de obtención de información son comúnmente "la información, la revisión documental, los vestigios y objetos reales, así como en algunos casos personas que tuvieron relación directa con los hechos estudiados o quienes, aunque no tuvieron relación directa con esos hechos, cuentan con información válida y confiable" (p.111).

Esta investigación es de carácter histórica porque se planteó como objetivo caracterizar los distintos juegos en papel presentes en las culturas escolares de generaciones de estudiantes de distintas épocas que crecieron en diversos contextos sociales, políticos, económicos y c culturales, por lo tanto, es una reconstrucción histórica que se apoyó de la metodología de la historia oral, particularmente a través de talleres y entrevistas a sus protagonistas.

\section{Método de investigación}

La metodología de la historia oral es de gran importancia para"reconstruir procesos socio-históricos a partir de la percepción y concepción de los protagonistas, convirtiéndose el testimonio oral en un nuevo documento escrito" (Lara y Antúnez, 2014); asimismo, Iño Daza (2018) argumenta que esta metodología de investigación cualitativa "al escuchar, reconocer las voces y dar voz a los sin voz a los marginados, su ámbito de estudio se focaliza en la memoria y el informante" (p.102). Con base en esto, cabe afirmar que la investigación utilizó la metodología de la historia oral para rescatar los elementos culturales relacionados con los juegos en papel en el contexto escolar.

\section{Informantes claves y técnicas de investigación}

Esta investigación fue desarrollada por profesores Universitario y de Educación Secundaria, ambos basados en las ideas que la docencia y la investigación son elementos indispensables para generar nuevos saberes desde las prácticas pedagógicas. En este sentido, los informantes claves fueron seleccionados a través del muestreo no probabilístico de carácter intencional (Arias, 2012), estableciendo como único criterio que estos fuesen grupos de clases asignados al docente durante el I semestre del curso 2021 .

Esto conllevó a trabajar con los siguientes informantes: de Educación Secundaria participaron los grupos de clase de estudiantes de IV y V año del Instituto Carlos Fonseca Amador del municipio de Cinco Pinos, departamento de Chinandega; y de Educación Superior, participaron los estudiantes de I año de la carrera Educación Musical, y los estudiantes de I y IV año de la carrera Ciencias Sociales, ambas carreras adscritas a la Facultad de Educación e Idiomas de la Universidad Nacional Autónoma de Nicaragua, Managua (UNAN-Managua). 


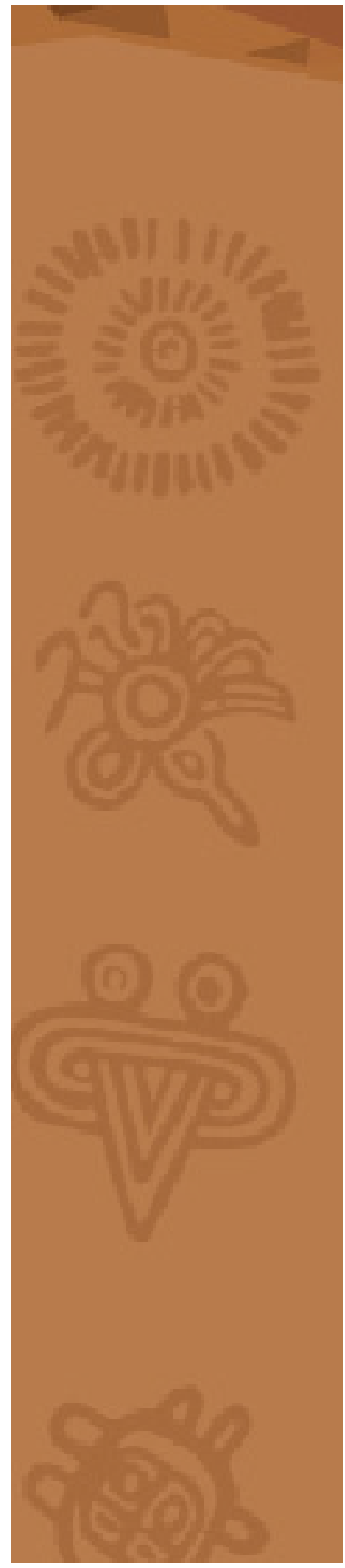

Cabe mencionar que, por razones de ética en la investigación, se ha recurrido a la confidencialidad de las identidades de los informantes claves, optando por utilizar seudónimos, esto basado en lo que propone Abreu (2017): "es indispensable resguardar su integridad física y mental para eso es necesario no divulgar en algunos casos la fuente o nombres particulares de las personas que suministraron una determinada información" (p.343).

La técnica de investigación que se utilizó para indagar acerca de los juegos en papel fue a través de talleres. Los talleres en la metodología de la historia oral son oportunos para reconstruir la historia de un pueblo, barrio o de acontecimientos. En esta modalidad habrá un coordinador, este propone los temas al grupo y anticipadamente estudia la temática a investigar, además, deberá mantener la discusión centrada en el grupo para generar interés y participación, organizar el ritmo de trabajo y sintetizar los aportes para estimular nuevas elaboraciones (Barela, Miguez y García, 2009), en este caso, se llevaron a cabo cinco talleres con los respectivos grupos de clases mencionados anteriormente, y los coordinadores de talleres fueron los docentes investigadores.

Así también, para alcanzar el cuarto objetivo de la investigación que fue precisar los usos didácticos de los juegos en papel en los procesos educativos, se procedió a diseñar una encuesta a estudiantes y una entrevista a profesores de Educación Primaria, esto basado en los requerimientos metodológicos propuestos en Bernal (2010) y Arias (2012). La finalidad de estos instrumentos de investigación fue construir desde la perspectiva de los profesores y de los estudiantes, el valor didáctico que tienen los juegos en papel en los procesos de enseñanza y aprendizaje en la escuela Primaria.

\section{RESULTADOS}

El ahorcado es un juego que se desarrolla en parejas en donde cada participante tiene un rol distinto: (1) El que ejecuta el juego, o (2) El adivinador. Para ello, el que ejecuta el juego debe seleccionar en su mente una palabra y trazar las cantidades de líneas en función de la cantidad de grafemas que componen la palabra seleccionada. El juego consiste en que el adivinador debe ir pronunciando letras deliberadamente, esto con el propósito de atinar a la palabra que el director del juego ha seleccionado. A medida que el adivinador se equivoque, el otro participante iniciará el dibujo esquemático de un muñeco(a), una cuerda y un árbol, hasta proceder a ejecutar al jugador en forma simbólica. Este juego permite la concentración, la formación de palabras, mejorar la ortografía y sobre todo mantienen motivado al jugador (González, 2021; García, 2021).

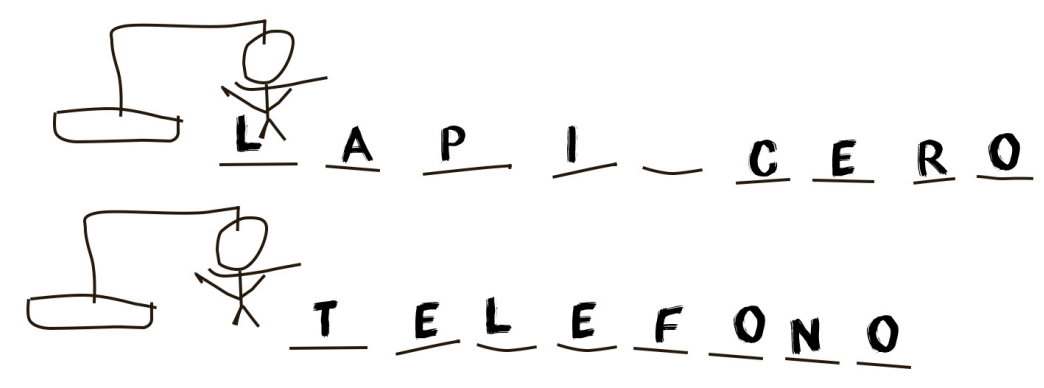




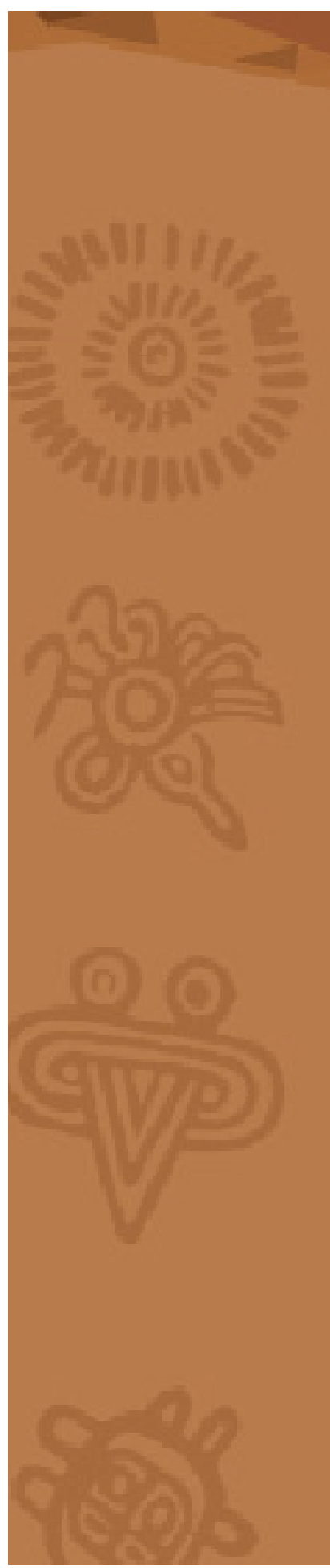

Stop es uno de los juegos en papel y lápiz más sencillos de desarrollar, se requiere una hoja con trazos en columnas con diferentes elementos que pueden estar determinados por los jugadores, incluye: Nombre, Apellido, Ciudad, Cosa, Color, Fruta, Animal; mismos a los cuales los jugadores le asignan de 5 a 20 puntos por indicador según estos convengan. El juego inicia cuando uno de los participantes recita la frase "con la letra...", es decir, se selecciona una letra y a partir de ese momento en el juego se inicia una competencia de agilidad mental para escribir palabras que inicien con la letra seleccionada en cada indicador.

El tiempo del juego está determinado por la capacidad de sus jugadores y termina cuando uno de los participantes ha completado todos los indicadores y pronuncia la palabra "stop". Posteriormente, el participante que terminó primero hace una suma de puntaje de cada uno de los jugadores, cabe mencionar que el ponderado de cada sujeto está determinado por las siguientes reglas: cuando se dijo Stop todos los participantes deben de dejar de escribir, si hay igualdad en una palabra el valor de los puntos disminuye a la mitad. Lo más importante es el desarrollo de habilidades motoras y cognitiva, y por si solo ofrece el desarrollo de competencias integrales (Castañeda, 2021; Rivas, 2021).

\section{El Rayos del sol}

Este juego es de dos participantes y para jugarlo únicamente se requiere lápiz, papel y tres objetos pequeños del medio, ya sea piedra, granos de maíz, frijoles o trigo, tapones o pedacitos de papeles arrugados. El juego inicia cuando los participantes deciden con qué objeto jugar y quién será el primero en colocarlo al centro, después cada jugador va colocando el resto de objeto y moviendo las piezas de manera estratégica con la pretensión de que tres piezas queden en el mimo rayo o en la misma dirección, ya que esta es la condición dado para que uno de los participantes gane el juego (Olivares, 2021; Ruiz, 2021).

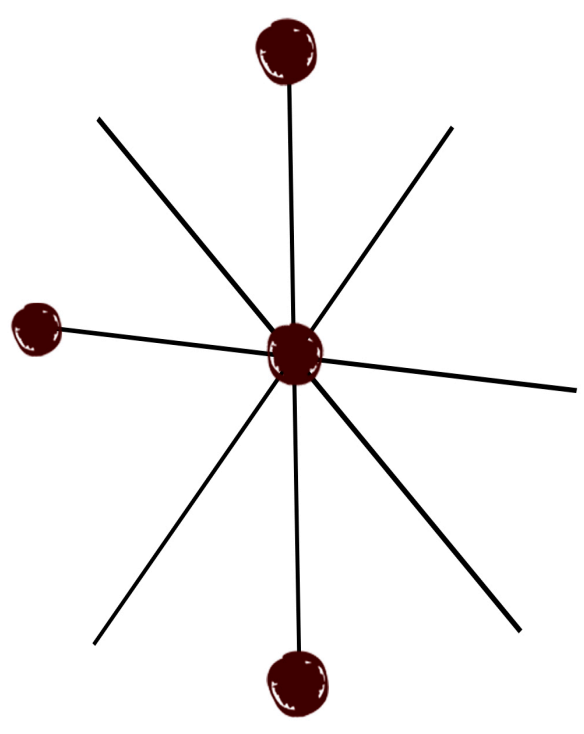




\section{El camino del diablo}

Los requerimientos de este juego son dos jugadores que alternadamente intercambian turnos, y como recursos físicos únicamente se requiere de lápiz y papel. El juego inicia cuando uno de los participantes coloca un duplicado de los números del 1 al 20 sobre una hoja en blanco de manera desordenada, ocupando así todo el espacio del papel. Luego cada participante alternadamente deberá unir a través de un trazo los números homólogos. La regla del perdedor del juego está condicionada por aquel jugador que al trazar la línea toque alguna línea o número, de esta manera, este sujeto pierde el juego (Flores, 2021; Lucas, 2021).

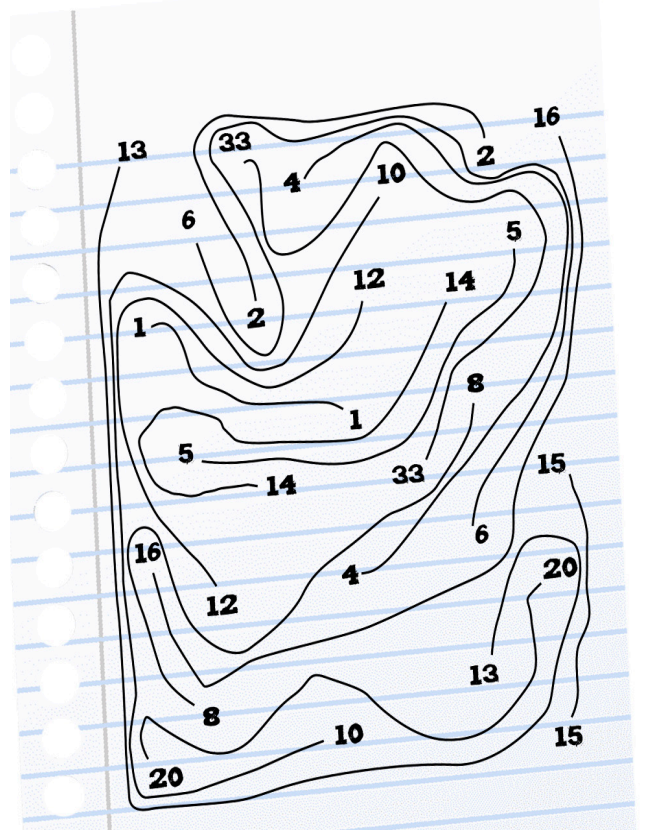

\section{Juego de la Z}

El juego de la Z no es muy común según los resultados obtenidos en los talleres. En este juego se necesita dos jugadores, varón y mujer, en la parte de arriba uno de los jugadores detalla la persona que le agrada o que le desagrada y las labores que le gustaría que realice, en la parte de abajo, el otro jugador indica partes del cuerpo y la persona que le agrada o le desagrada, al terminar se unen las palabras formando una $Z$, seguidamente se lee la oración completa dándole el lado más divertido al juego. Según la imagen que se presenta en este juego la primera oración seria: Rosa plancha la cabeza de Juan (Castañeda, 2021; Lucas, 2021)

\section{Juego de la Z}

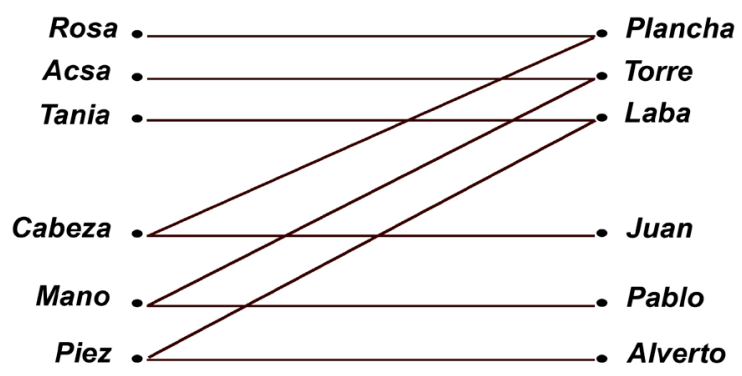




\section{Raíces}

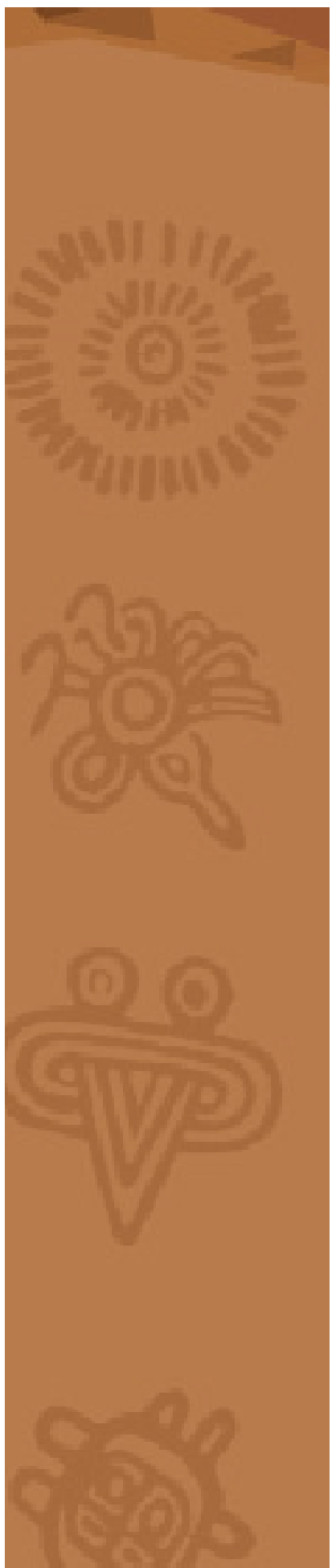

\section{Guerra naval}

La guerra naval es un juego que se desarrolla con lápiz y papel, preferiblemente en cuadernos cuadriculados. Este se lleva a cabo con la participación de dos personas que simulan una confrontación bélica a través de submarinos de guerra.

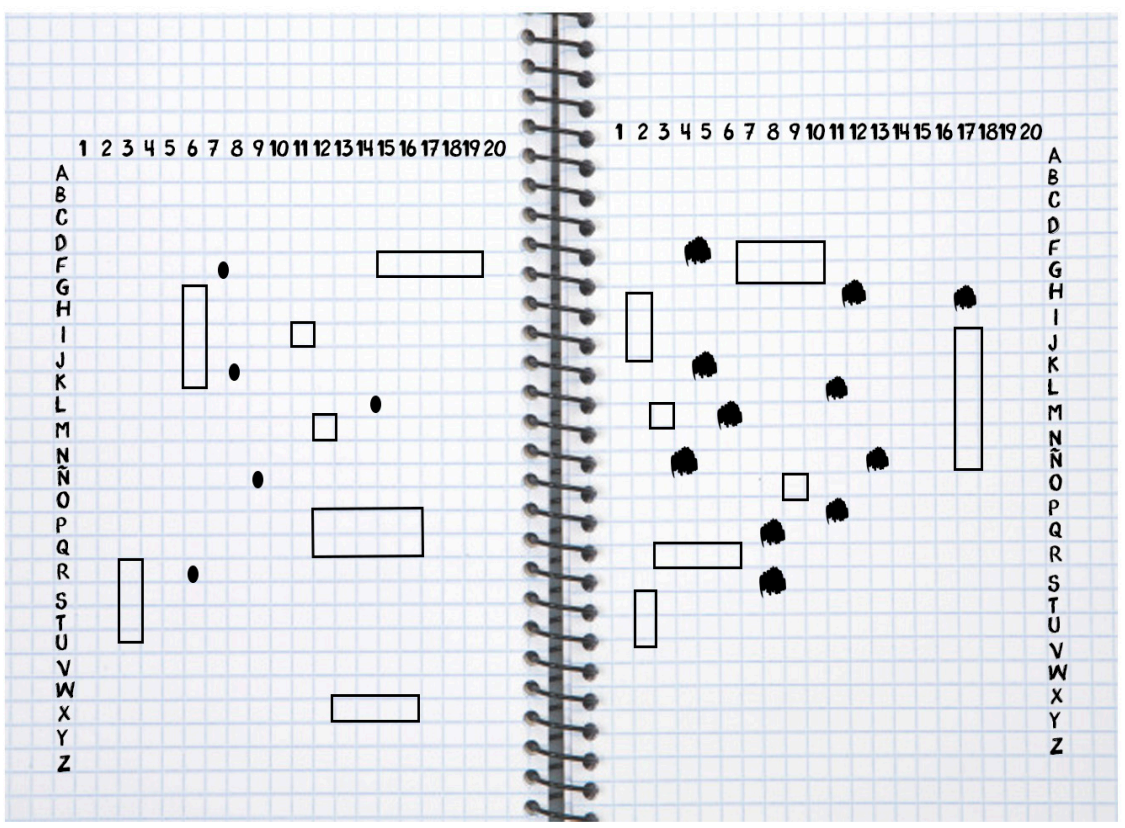

El juego inicia construyendo el área geográfica de la guerra, se deben colocar números del 1 al 15 en la parte superior de la hoja, asimismo, se deben escribir las primeras 15 letras del alfabeto en el margen izquierdo en sentido vertical y descendente. Luego, en el espacio del centro se deben dibujar, a preferencia de los jugadores, un barco de guerra grande, dos semi grandes medianos, dos medianos y dos pequeños, para un total de siete embarcaciones. Luego cada participante tendrá su turno para elegir un número y una letra (coordenadas) con el propósito de alcanzar la ubicación del barco. Si las coordenadas precisan la ubicación de los barcos, entonces ese contingente militar se destruirá o se debilitará, esto según los acuerdos que establezcan los participantes, y en caso de fallar, solamente se coloca un punto sobre las coordenadas señaladas (Olivares, 2021; Lucas, 2021). 


\section{Puntito}

Puntito es un juego de estrategia entre dos o más personas. El juego inicia cuando sobre una hoja de papel se dibujan la misma cantidad de puntos en sentido horizontal y vertical, a un espacio de una cuadrícula de cuaderno matemático. Luego los jugadores se alternan turnos para ir uniendo los puntos a través de rayas verticales y horizontales. La disputa se intensifica cuando uno de los jugadores encierra los puntos en forma de "caja", colocando la inicial de su nombre al interior de la misma, de esta manera, quien más cajas acumula, ese es el ganador del juego (González, 2021; Flores, 2021; García, 2021).

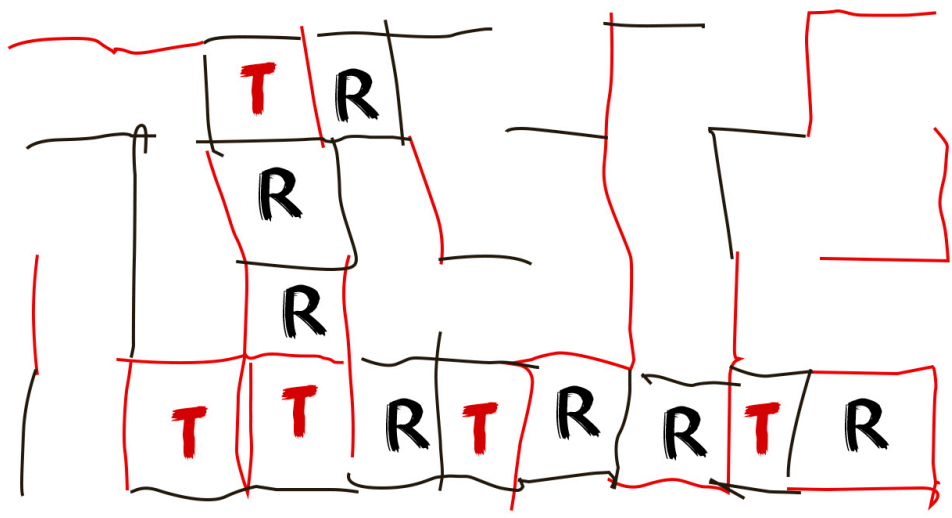

\section{Equis y Cero}

Tres en línea es el nombre oficial para X 0 (equis y cero). Es un juego de poca complejidad que requiere papel y lápiz, se juega en pareja, en el destaca la concentración y la habilidad de pensar en función al próximo movimiento de tu adversario. Las reglas son simples: se intercambia el inicio por pareja, gana el primero en colocar consecutivamente tres $\mathrm{X}$ o tres 0 de manera diagonal, horizontal o vertical, $\mathrm{y}$ el ganador debe trazar una línea en sus tres elementos; cabe mencionar que este juego es común que los jugadores empaten. Es un juego que los docentes pueden utilizar para liberar estrés en sus estudiantes y continuar con las asignaciones curriculares (González, 2021; Flores, 2021; García, 2021).
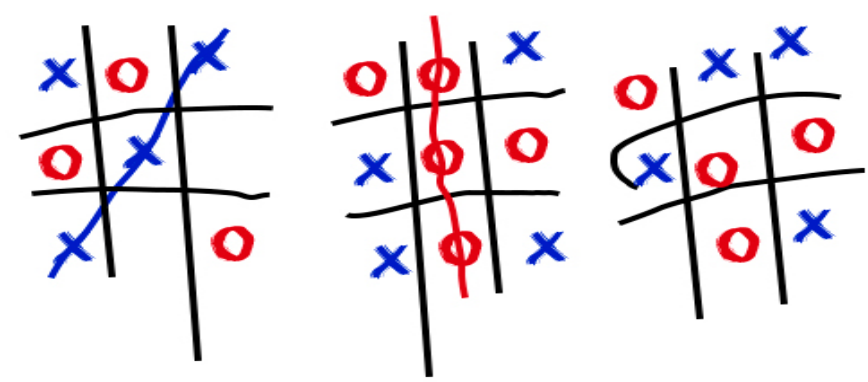

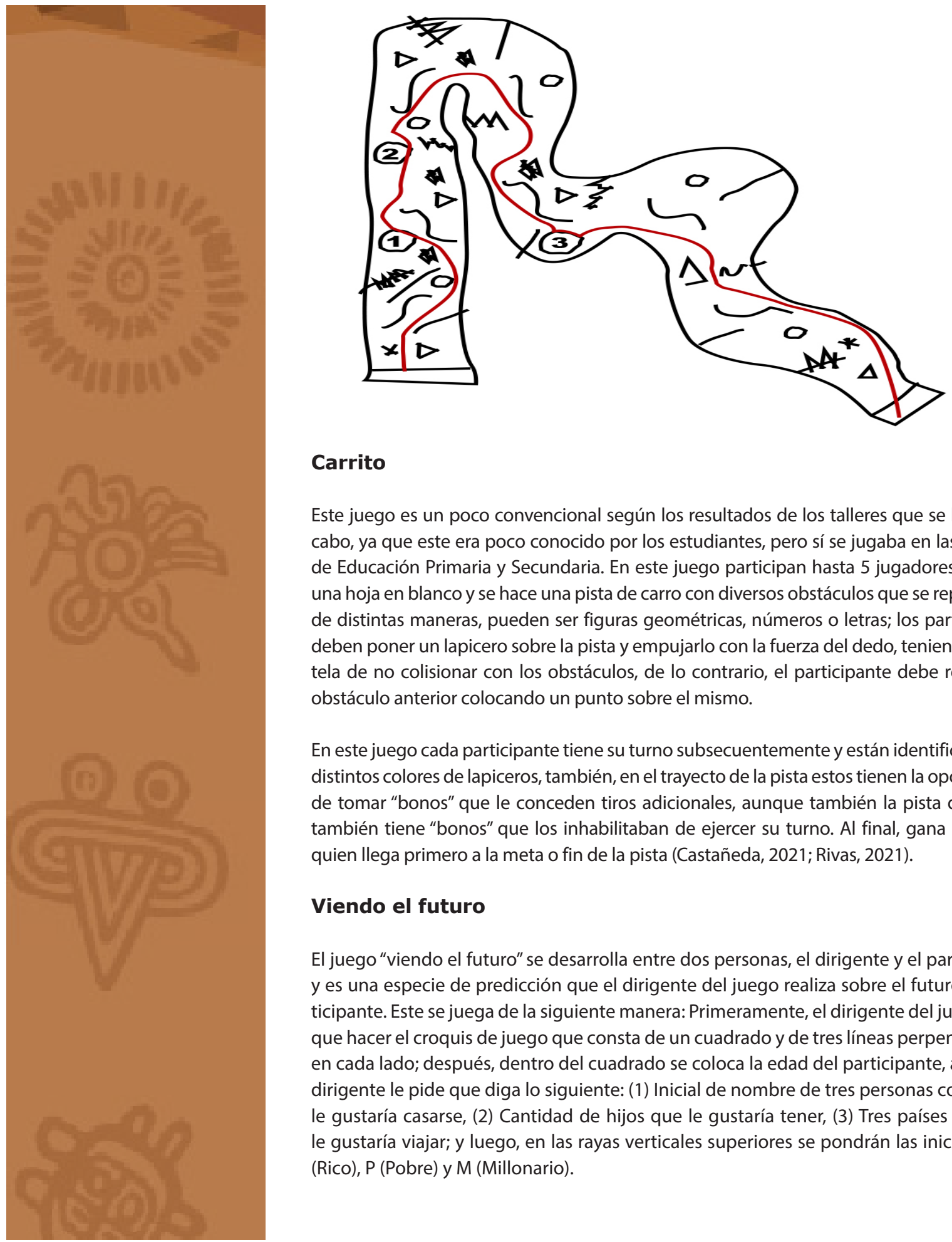

\section{Carrito}

Este juego es un poco convencional según los resultados de los talleres que se llevaron a cabo, ya que este era poco conocido por los estudiantes, pero sí se jugaba en las escuelas de Educación Primaria y Secundaria. En este juego participan hasta 5 jugadores, se toma una hoja en blanco y se hace una pista de carro con diversos obstáculos que se representan de distintas maneras, pueden ser figuras geométricas, números o letras; los participantes deben poner un lapicero sobre la pista y empujarlo con la fuerza del dedo, teniendo la cautela de no colisionar con los obstáculos, de lo contrario, el participante debe retornar al obstáculo anterior colocando un punto sobre el mismo.

En este juego cada participante tiene su turno subsecuentemente y están identificados con distintos colores de lapiceros, también, en el trayecto de la pista estos tienen la oportunidad de tomar "bonos" que le conceden tiros adicionales, aunque también la pista de carrera también tiene "bonos" que los inhabilitaban de ejercer su turno. Al final, gana la partida quien llega primero a la meta o fin de la pista (Castañeda, 2021; Rivas, 2021).

\section{Viendo el futuro}

El juego "viendo el futuro" se desarrolla entre dos personas, el dirigente y el participante, y es una especie de predicción que el dirigente del juego realiza sobre el futuro del participante. Este se juega de la siguiente manera: Primeramente, el dirigente del juego tiene que hacer el croquis de juego que consta de un cuadrado y de tres líneas perpendiculares en cada lado; después, dentro del cuadrado se coloca la edad del participante, a quien el dirigente le pide que diga lo siguiente: (1) Inicial de nombre de tres personas con las que le gustaría casarse, (2) Cantidad de hijos que le gustaría tener, (3) Tres países a los que le gustaría viajar; y luego, en las rayas verticales superiores se pondrán las iniciales de $R$ (Rico), P (Pobre) y M (Millonario). 
El juego se desarrolla haciendo un conteo sobre cada raya según el número de la edad del participante. A medida que se realiza el conteo, se van eliminando las opciones, hasta que al final queda una opción de cada casilla. En el ejemplo de la presente imagen se puede leer lo siguiente: El participante se casará con Leonor y tendrán un hijo, viajarán a España y serán millonarios (Lucas, Flores y Ruiz, 2021).

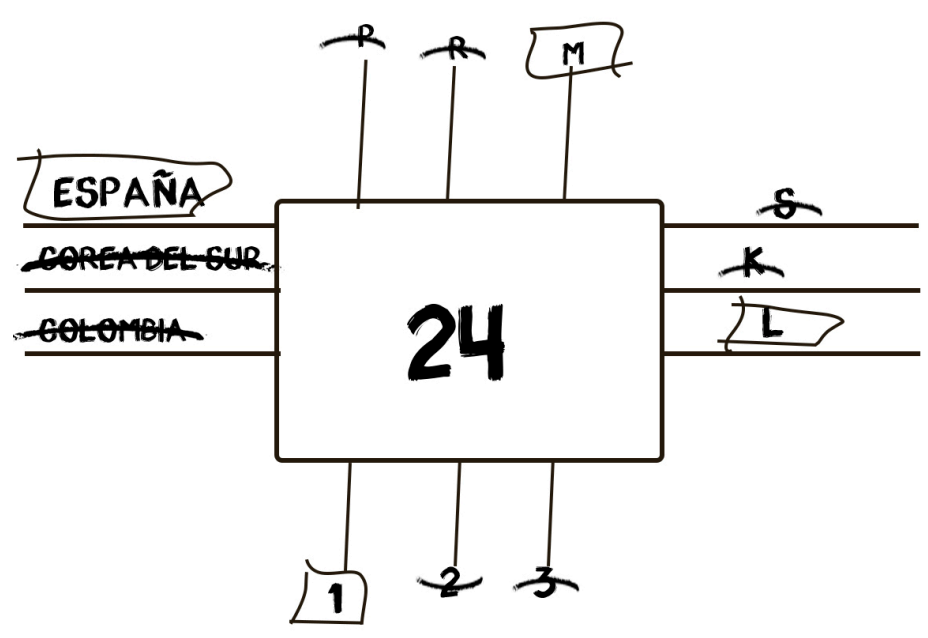

\section{Los juegos en papel en la cultura escolar}

En la cultura nicaragüense los juegos ocupan un lugar sobresaliente, el Yo imaginativo presente en nuestro imaginario colectivo ha conducido a decenas de generaciones a llevar a cabo las invenciones más creativas que pueden surgir en un contexto caracterizado por la escasez y la precariedad, sin embargo, el Ser fantasioso del nicaragüense ha posibilitado la apertura de nuevos espacios de esparcimiento y socialización que han venido construyendo rasgos culturales en los distintos tiempos.

Los juegos en papel precisamente han sido una manifestación de esta cultura surgida en los contextos escolares tradicionales de Nicaragua, de ahí que muchos recuerden a profesores ofuscados en el aula de clase arrebatando a los estudiantes hojas rayadas cuando estos jugaban sigilosamente sin prestar atención a su clase. Para dar continuidad a esto, se realizaron entrevistas para conocer las opiniones de los estudiantes acerca de los momentos en que practicaban estos juegos en papel. A continuación, sus respuestas:

Por lo general nosotros jugábamos estos juegos en el lapso intermedio de las clases (González, 2021)

Lo jugaba en clase cuando el maestro nos daba tiempo libre, cuando ya habiamos terminado las tareas, a veces también lo jugábamos para matar tiempo mientras venía el profesor de la otra clase (Lucas, 2021) 


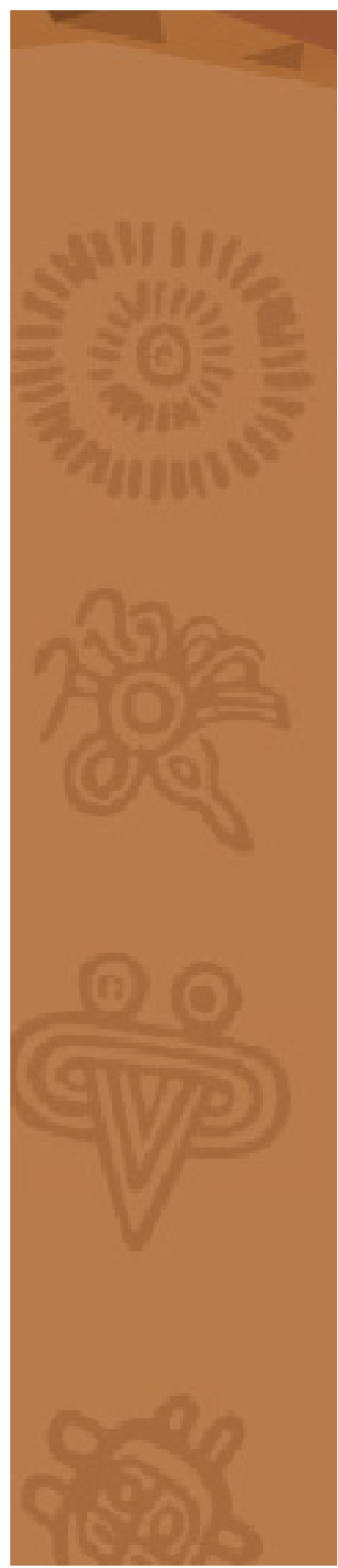

Jugábamos en nuestro tiempo libre, cuando el profesor no estaba o si no en el momento de la clase, cuando esta estaba aburrida (Flores, 2021)

Yo con mis amigas jugaba cuando estaba aburrida en la clase, para distraernos o para matar tiempo porque la clase estaba aburrida (García, 2021)

Jugábamos a la hora de entrada mientras esperábamos que llegara el profesor, en las horas libres, a la hora de recreo o cuando no llegaba la maestra o el maestro (Ruiz, 2021)

\section{El uso didáctico de los juegos en papel en los procesos edu- cativos}

Juegos en papel y lápiz, es una estrategia didáctica que obedece al método lúdico donde los estudiantes son los protagonistas de su aprendizaje. El juego se convierte en el medio para relacionarse con su entorno y este a su vez permite el desarrollo de la creatividad incrementando sus conocimientos.

Los diferentes juegos en papel como: puntos y cajas, laberintos, sopa de letra, el ahorcado, stop; por mencionar algunos, son de gran utilidad en los procesos de enseñanza y aprendizajes, estos mismos se pueden aplicar en los diferentes niveles de formación Primaria, Secundaria y, ¿Por qué no a nivel Universitario, sin importar el área de conocimiento?

Aprender también puede ser divertido con esta estrategia aprovechando las bondades que ofrecen estos juegos y la familiaridad que sienten los estudiantes con ellos. Bastaría con preguntarnos ¿Quién de nosotros no conoce uno de ellos? Ya que a través de los tiempos siempre se juegan de una u otra manera, puesto que esto es parte de una cultura del entretenimiento o pasatiempo sano.

En uno de los centros educativos de Matagalpa se aplicó una encuesta a estudiantes de primero a quinto grado para corroborar el conocimiento que tienen en referencia a los juegos en papel, y acerca de qué piensan sobre su uso en los procesos de formación. Los resultados obtenidos indican que los juegos que más reconocen este grupo de estudiantes son: sopa de letras, Stop, tres en raya o bien conocido como X 0, crucigrama. También fue necesario saber que, si ellos consideran de utilidad estos juegos para sus aprendizajes, y sin duda alguna manifestaron un rotundo sí. Es decir, los diferentes juegos pueden integrarse sin exclusión alguna a todas las asignaturas.

Como parte de esta temática se consultó a docentes de diferentes grados de primaria, quienes manifiestan conocer los juegos presentados e indican a su vez que son de utilidad para los estudiantes ya que desarrollan motivación, interés por aprender, seguridad, lógica, y orden ya que cada juego conlleva reglas a seguir. No obstante, en los juegos podemos destacar bondades: estimula la atención, aceptación ante situaciones que no los favorecen (ganar o perder), motivación, interés, sentido de pertenencia en lo que hacen, mejoran su psicomotricidad, concentración. 
Toda la comunidad educativa reconoce las bondades de los juegos en papel para el aprendizaje, entonces ¿Qué falta para integrarlo? Será más de esfuerzo por los docentes, ver el aprendizaje como una actividad divertida y con mayor significado, y despojarnos de mitos y concepciones erróneas que interpretan el juego como un ocio solamente, por el contrario, los juegos son una herramienta lúdica que utilizados con fines didácticos facilitan el alcance de las competencias requeridas por los estudiantes de grado.

En fin, la construcción del conocimiento se logra cuando se retoman los métodos, estrategias y actividades que atrapan la atención y mantienen el interés de los estudiantes, retomando juegos sencillos con recursos asequibles a cada uno, como lo son los juegos en papel y lápiz, se podría aportar al aprendizaje de forma significativa, por lo tanto, esto es un reto que se puede incorporar en el quehacer de los docentes.

\section{CONCLUSIONES}

Los juegos son una manifestación cultural presente en todos los contextos de la vida y, siendo la escuela un espacio de interacción y socialización permanente entre las personas, esta ha sido un sitio propicio para la construcción de prácticas culturales al margen de los procesos de formación ciudadana. Esto deja consigo un enorme reto para la investigación en ciencias sociales y para la investigación educativa, puesto que amplía las nociones estrictas que sitúan a la escuela únicamente como formadora y la coloca como centro de construcción de cultura y de nuevas relaciones interpersonales.

La investigación social llevada a cabo ha rescatado diez juegos tradicionales en papel presentes en las culturas escolares nicaragüenses, se ha descrito cada juego, se ha precisado en qué momentos se jugaban y se ha hecho énfasis en el valor educativo que estos representan. En este sentido, este proceso investigativo rescata el valor cultural y la optimización educativo que pueden tener los juegos como el ahorcado, el camino del diablo, rayos del sol, stop, juego de la Z, guerra naval, puntito, equis y Cero, carrito y viendo el futuro.

El reto de educadores e investigadores sobre el espectro educativo es amplio y emergente. La modernidad nos impone hoy más que nunca la imperante necesidad de rescatar todas aquellas manifestaciones culturales que con el pasar del tiempo han ido quedando relegadas en nuestra cotidianidad. El hecho educativo es objeto de estudio de la pedagogía y sus didácticas, pero también es un punto de interrelación entre las diversas ciencias de la educación y las distintas disciplinas sociales, quienes, a través de procesos investigativos integrados, seguramente proporcionarán densos, valiosos y oportunos hallazgos investigativos que aportarán a la reconstrucción de las realidades que se viven en las escuelas. 


\section{Raíces}

Revista Nicaragüense de Antropología Año 5 No.9| 2021 Enero - Junio

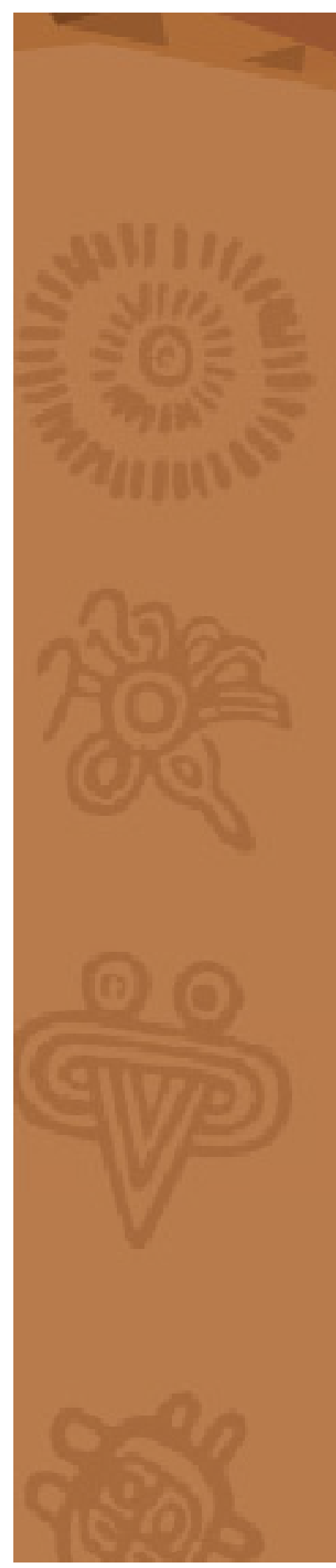

\section{REFERENCIAS BIBLIOGRÁFICAS}

Abreu,A.(2017,julio).LaÉtica en la InvestigaciónEducativa. Revista Scientific,2(4):338-350.https//doi.org/10.29394/scientifici.isn.2542-2987.2017.24.19.338350

Ander-Egg, E. (2000). Metodología y práctica delaAnimación Sociocultural. Madrid:Plaza.

Arias, F.(2012). El proyectode investigación. Introducción a la metodología científica. (6taed). Episteme.

Barela, L, Miguez, M.y García, L. (2009).Algunos apuntes sobre historia oral y cómo abordarla: Dirección General Patrimonio e Instituto Históico

Bemal, C. (2010).Metodología dela investigación. (3raed).PearsonEducación.

Cuz,A.Díaz,A.yHemández, Y.(2020,julio).Loscastigosescolaresen ladécadadelos setenta. Relatosdeestudiantes y profesoress. Revista HumanismoyCambio Social,(16):12-25.https//doi.org/10.5377hes.Wi16.10452

Díaz,A.,Arauz, M, Sandoval,A.y Rizo,M. (2019,julio).Promoviendolosjuegostradicionalesnicaragüenses. Unaexperiencia de animación sociocultural. Raíces, (6):71-83.https//doi.org/10.5377/raices.v3i6.9031

Iño Daza, W. (2018). Investigacióneducativa desde un enfoque cualitativo: la historiaoral comométodo. Voces de la Educación, 3(6):93-110. hthps//dialnetunirioja. es/servlet/articulo?codigo-6521971

Lara, P. y Antúnez, A. (2014, diciembre). La historia oral como altemativa metodológica para las ciencias sociales. Revista de Teoría y Didáctica de las Ciencias Sociales, (20):45-62.https:/www.redalyc.orgpdff652/65247751003.pdf

\section{REFERENCIAS DE ENTREVISTAS}

Castañeda, Luis. (2021, 17 de abril). Entrevista de A, Díaz Pérez [Cinta de audio]. Proyecto Los juegos en papel en las culturas escolares nicaragüense. Managua, Nicaragua.

Flores, Aldo. (2021, 06 de abril). Entrevista de A, Díaz Pérez [Cinta de audio]. Proyecto Los juegos en papel en las culturas escolares nicaragiense. Managua, Nicaragua.

García, Gema. (2021, 06 de abril). Entrevista de A, Díaz Pérez [Cinta de audio]. Proyecto Los juegos en papel en las culturas escolares nicaraguiense. Managua, Nicaragua.

González, Elvi. (2021, 15 de abril). Entrevista deA, López, [Cinta de audio]. Proyecto Los juegos en papel en las culturas escolares nicaragüense. Managua, Nicaragua.

Lucas, Naomi. (2021, 06 de abril). Entrevista de Y, Hemández. [Cinta de audio]. Proyecto Los juegos en papel en las culturas escolares nicaragiense. Managua, Nicaragua.

Olivares, Francisco.(2021, 15 deabril).EntrevistadeA, DízzPérz [Cinta deaudio].Proyecto Losjuegos en papel en las culturas escolares nicaragüense.Managua, Nicaragua.

Rivas, Daniela. (2021, 17 de abril). Entrevista de A, Díaz Pérez [Cinta de audio]. Proyecto Los juegos en papel en las culturas escolares nicaraguiense. Managua, Nicaragua.

Ruiz, Francis. (2021,15 deabil). Entrevista deA, Díaz Pérz [Cinta deaudio]. ProyectoLos juegos en papel en las culturas escolares nicaragüense. Managua, Nicaragua. 
Adolfo Alejandro Díaz Pérez

Licenciado en Ciencias de la Educación con mención en Ciencias Sociales en la Universidad Nacional Autónoma de Nicaragua, Managua (UNAN-Managua) y máster en Educación e Intervención Social. Ha participado como ponente en congresos nacionales e internacionales y recientemente ha realizado publicaciones en revistas de educación, entre ellas: Nuevos ambientes educativos en el aprendizaje de las Ciencias Sociales. Sistematización de una experiencia didáctica en Educación Secundaria en Nicaragua; además, de publicaciones en diarios como: En septiembre Rigoberto dijo ¡Patria! Actualmente es docente de la carrera Ciencias Sociales de la Facultad de Educación e Idiomas de la UNAN-Managua, y estudia el Doctorado en Educación e Intervención Sociales en la Facultad Regional Multidisciplinaria de Chontales (FAREM-Chontales) de la UNAN-Managua.

Arlen Idania López Delgado

Licenciada en Pedagogía con mención en Educación Primaria en la Universidad Nacional Autónoma de Nicaragua, Managua (UNAN-Managua) y máster en Educación Comparada en UNAN-León. Actualmente es Ejecutiva de Posgrado y Educación Continua UNAN-Managua en la actualidad y docente del departamento de Pedagogía en el área curricular, didáctica y formación docente. Ha participado en Congresos, Foros y otros eventos académicos a nivel nacional en el tema de Formación de Docente, Educación Superior y Continuidad Educativa, asimismo, participó en la Transformación Curricular del Ministerio de Educación (MINED) 2015-2017, y cuenta con experiencia en la elaboración de diseños curriculares de grado y posgrado.

\section{Yesika Karina Hernández Navarro}

Licenciada en Ciencias de la Educación con mención en Ciencias Sociales en la Universidad Nacional Autónoma de Nicaragua, Managua (UNAN-Managua) y estudiante de maestría en Educación con énfasis Didáctica de las Ciencias Sociales por la UNAN-Managua. Ha realizado publicaciones científicas asociadas al área socioeducativa, entre ellas: Los castigos escolares en la década de los setenta. Relatos de estudiantes y profesores. Actualmente ejerce la investigación educativa, y se desempeña como profesora de Educación Secundaria en el Instituto Carlos Fonseca Amador del municipio de Cinco Pinos, departamento de Chinandega. 